University of Nebraska - Lincoln

DigitalCommons@University of Nebraska - Lincoln

Faculty Publications in Computer \& Electronics Electrical \& Computer Engineering, Department Engineering (to 2015)

2007

\title{
Collaborative Image Transmissions Based on Region and Path Diversity in Wireless Sensor Network
}

\author{
Honggang Wang \\ University of Nebraska-Lincoln \\ Dongming Peng \\ University of Nebraska-Lincoln, dpeng2@unl.edu \\ Wei Wang \\ University of Nebraska-Lincoln \\ Hamid Sharif \\ University of Nebraska-Lincoln, hsharif@unl.edu \\ Hsiao-Hwa Chen \\ National Sun Yat-Sen University, Taiwan
}

Follow this and additional works at: https://digitalcommons.unl.edu/computerelectronicfacpub

Part of the Computer Engineering Commons

\footnotetext{
Wang, Honggang; Peng, Dongming; Wang, Wei; Sharif, Hamid; and Chen, Hsiao-Hwa, "Collaborative Image Transmissions Based on Region and Path Diversity in Wireless Sensor Network" (2007). Faculty Publications in Computer \& Electronics Engineering (to 2015). 2.

https://digitalcommons.unl.edu/computerelectronicfacpub/2
}

This Article is brought to you for free and open access by the Electrical \& Computer Engineering, Department of at DigitalCommons@University of Nebraska - Lincoln. It has been accepted for inclusion in Faculty Publications in Computer \& Electronics Engineering (to 2015) by an authorized administrator of DigitalCommons@University of Nebraska - Lincoln. 


\section{Collaborative Image Transmissions Based on Region and Path Diversity in Wireless Sensor Network}

\author{
Honggang Wang,Dongming Peng,Wei Wang,Hamid Sharif \\ University of Nebraska Lincoln, Omaha, USA \\ \{hwang, dpeng, wwang, hsharif\}@unlnotes.unl.edu
}

\author{
Hsiao-Hwa Chen \\ National Sun Yat-Sen University, Taiwan \\ hshwchen@mail.nsysu.edu.tw
}

\begin{abstract}
Many Sensor applications such as monitoring and surveillance may require image sensor array to conduct collaborative image transmissions in Wireless Sensor Networks (WSN). The large size image transmissions cause bottlenecks in WSN due to the limited energy resources and network capacity. In this paper, we propose a collaborative transmission scheme for image sensors to utilize inter-sensor correlations to decide transmission patterns based on transmission path diversities, which achieves minimal energy consumption, balanced sensor lifetime and required image quality. This optimization scheme not only allows each image sensor to transmit optimal fractions of the overlapped images through appropriate transmission paths in energy-efficient way, but also provides unequal protection on the overlap image regions through path selections and resource allocations to achieve good transmission image quality. The simulation results show that the proposed image transmission scheme can achieve considerable gains in terms of the network lifetime extension, image distortion reduction, and energy efficiency.
\end{abstract}

\section{INTRODUCTION}

Many applications, e.g., multimedia surveillance networks, target tracking, environmental monitoring, and traffic management systems, require effective harvesting and communication of event features in the form of multimedia such as image, and video. They require energy-efficient multimedia processing and may need image sensor array to conduct collaborative image transmissions under limited sensor resource constraints. However, image sensors generate a large amount of data loads in WSN, which become the bottlenecks of network transmission. These burdensome image data transmissions in WSN can drastically degrade the network performance and network lifetime due to the limited power in the sensors and relay nodes [1]. How to tackle this problem is still an open and on-going research topic. Most existing image compression methods only take advantage of the intra-image data redundancy, which is in a single sensor's measurement; on the other hand, although there may exist large volumes of data redundancy among the sensed images in the sensor array, a comprehensive joint image compression scenario for the images collected by the correlated sensors would be impractical, unless these image data reach an aggregation point. This is because such joint compression would require the simultaneous availability of image data from multiple sensors, involving high communication overhead for comprehensive data exchange. Therefore, it is difficult to increase the communication energy efficiency of correlated image sensors and extend the overall WSN lifetime. The redundancy of data transmission among correlated image sensors could not be easily exploited to save communication energy through common image compression methods.

In recent literature, the WSN research in utilizing sensor correlation is focused on either cooperative methods [2] or predictive methods [3]- [5].The former heavily involve intersensor communication overhead, which could be prohibitively high in the case of image applications. The latter must use prior knowledge of the sensor constellation. There have been reports on Distributed Source Coding (DSC) [5], applied onto multiple source sensors in WSN for efficient coding without mutual data exchange while the redundancy can be removed. However, DSC has major challenges particularly in image applications due to the difficult image correlation modelling, synchronization, etc. In addition, how to utilize the sensor correlation model for efficient image transmissions should not only be determined with the consideration of source image sensors themselves, but also be guided with the network parameters such as the routing pattern.

In this paper, we propose an effective approach where the data redundancy among correlated image sensors can be considerably reduced by a simple scenario, different from either joint image coding or distributed source coding. The communication overhead for data exchange is relatively small to exploit the correlations in the proposed approach. Furthermore, we investigate and consider both source sensor image transmission schemes and routing path selection together in WSN. We assume that image sensors are installed in various locations of a large size sensor network to monitor or track objects. These sensed images may overlap with each other. The sensors have limited capability and resource to process the images; therefore, images have to be sent to sink (or cluster head) for more sophisticated processing. As the sink may be a little far away from the image sensor, the images then have to be transmitted by multiple hops through relay sensors to the sink. It requires an effective image transmission pattern, working with energy-ware routing strategy, to handle largesize image data.

Further, it would be very important to exploit the intercorrelation of these sensed images of sensors with consideration of image region diversities (i.e., different image region importance levels among the overlap and non-overlap regions 
of the sensed images). This kind of region diversities may be utilized in multiple routing path transmissions in order to achieve higher energy efficiency, longer network life time and required image quality. For example, different routing path in network can have different reliability level and power consumption, transmitting most of image packet on reliable path might result in unnecessary power consumption. The question is how to allocate these diverse image regions to different paths so that for a total image distortion, the energy is minimized and vice versa.

\section{IMAGE REGION DIVERSITY OF MULTIPLE SENSORS}

In our research, a heterogeneous cluster-based wireless Image Sensor Network architecture is assumed.The clustered image sensor networks include the single hop and multi-hop scenarios. A single hop scenario is the one in which sensor nodes use single hopping to reach the cluster head $(\mathrm{CH})$. In a multi-hop scenario, several images sensors use multi-hopping to reach the cluster head. For example, three image sensors within one cluster might transmit their captured image by 1 , 2 or 3 hops separately. To be practical, we assume maximum hops from image sensors to cluster head is not too high $(<5$ hops). With the clustered-based image sensor network, we are interested in collaborative image transmission from image sensors to cluster head either by one hop or multiple hops. The efficient communication strategy between cluster heads is beyond our discussions in this paper and can be solved by many existing solutions. Based on single hop or multi-hop scenarios, given a random deployment of image sensors and relay sensors to cluster heads along with the corresponding energy on each sensor as shown in Figure 1(b), we want to find an optimal transmission pattern with respect to single hop or multiple hop paths to the cluster head.In Figure 1(b), it gives a part of cluster-based network topology on which we focus our study, and several coordinated image sensors transmit their captured image to the cluster head either by single hop or multiple hops to the cluster head. For simplicity yet without loosing generality, we list the following assumptions: Each image sensor sends non-overlapping region and possibly part of overlapping regions shared with other image sensors within its field of view; The union of non-overlap regions and overlap regions equals the whole area of view for all correlated image sensors. Figure 1(b) shows a generic sensor network model with three image sensors capturing pictures F1, F2 and F3. In Figure 1(b), F1, F2 and F3 are three images taken by three image sensors, respectively. Each image can be separated into Overlap (OVL) regions and Non-Overlap ( $\left.N_{-} O V L\right)$ region as illustrated in Figure 1(a). This scenario is very typical in image sensor-based network due to random deployment of sensors with highly random camera angle and applicationinitiated collaborative detection of target. Overlap area is the intersection of the fields of image captured by each cameras array. Non-overlap region is the each image region not being included in the overlap region. Each image sensor will transmit its N_OVL region and part of OVL regions to the cluster head via single-hop or multi-hop paths. To save communication energy, it would be important for each source sensor to send its own N_OVL region and not to send the portion of OVL region that has already been sent by another source sensor who shares this portion of OVL region. We use $x_{i, j}$ to denote the fraction of the overlap region $O V L_{j}$ that is to be sent by sensor $i$. All $x_{i, j}$ form a matrix $X$ called distribution ratio of OVL region transmissions. Therefore, each sensor $i$ will send $N_{-} O V L_{i}+\sum_{j=1}^{M} x_{i, j} \cdot O V L_{j}$ amounts of image data.

In addition, there is image region diversity for image transmissions due to following two reasons: In most applications, all OVL regions should be the major fields of interest; The transmission quality of an OVL region is dependent on more than one source sensors because each of them may send a portion of this OVL region. Therefore, it is necessary to provide unequal protection to transmit OVL and N_OVL image regions and achieve expected image quality. This kind of diversity would potentially provide energy efficient image transmission over multiple paths.

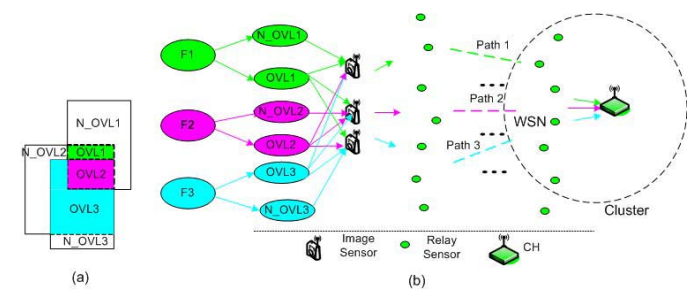

Fig. 1. Sensor network model of image transmission

\section{RESOURCE-DISTORTION AND DISTRIBUTION RATIO ANALYSIS}

As we mentioned, image transmission over wireless sensor networks operates under a set of unique resource constraints. It is expected that the amount of resource supply of the sensor nodes determines the image quality of service that one can expect. For a given configuration of system resources on each route paths(such as rate, power supply) and distribution ratio of OVL image regions, we are interested in what level of image quality we would be able to achieve with minimized energy consumptions.

The natural image is generally delivered by layer bit stream. There are different fault-tolerance levels for disturbance on the compressed image bits to be transmitted in wireless networks, particularly wireless sensor network environment. With consideration of the distortion reduction at each layer, we form a performance metric for the overall image sensor transmissions. The following expression gives the image distortion reduction:

$$
E\left(\Delta_{p}\right)=\sum_{j=1}^{M}\left(\begin{array}{l}
\sum_{i=1}^{j} \Delta_{i} \cdot\left(\prod_{i=1}^{j}(1-\overline{P E R}(p, i))\right) \\
\cdot \overline{P E R}(p, i+1)
\end{array}\right)
$$

Where $E\left(\Delta_{p}\right)$ is average distortion reduction and $\overline{P E R}(p, i)$ denotes average packet loss probability for the $i$-th layer packet on path $p$, and $\Delta_{i}$ is the distortion reduction 
associated with the $i^{\text {th }}$ layer, $M$ and are the total number of transmitted layer packets of image regions. Details about distortion reduction can be referred from recent studies in [6]. In (1), $\overline{P E R}$ is average packet loss probability determined by the transmission paths and channel characteristics (BER, ARQ, Power, Rate, etc.), it can be modelled as described in [6][8].Therefore, packets being transmitted over multiple paths with diversities such as BER, maximum retransmission count, hop counts, packet length would have different packet loss probability. Those diversities work with different distribution ratio $X$ will make different image quality transmissions. For example, assume that $\left\{\begin{array}{l}x_{11}, x_{12} \\ x_{21}, x_{22}\end{array}\right\}$ is the distribute ratios of $\{$ OVL1, OVL2 $\}$ image regions that two image sensors (sensor 1 and sensor 2) have to transmit. Therefore, image sensor 1 will transmit $x 11 \cdot O V L 1+x 12 \cdot O V L 2$ image and image sensor 2 will transmit $(1-x 11) \cdot O V L 1+(1-x 12) \cdot O V L 2$ $(x 21=1-x 11, x 22=1-x 12)$. As shown in Figure 2(b), we can observe different distribution ratio will cause different image transmission quality even at the same BER value. At the same time, the higher image quality could be achieved by the distribution ratio $(x 11=0.2, \times 12=0.3)$. Therefore, transmission distribution ratio combinations could impact performance of image transmission under different path diversity. Sensed image is based on figure 2(a). Further, in order to achieve required image quality, corresponding desirable BER at each link on the multiple hop paths has to be guaranteed. Dynamic changing transmission power and rate is an effective way to assure desirable BER. Figure 3 provides a solution illustration with Unequal Path protection (UPP) scheme by path selections and Unequal BER protection (UBP) scheme implemented by resource allocations(allocate power and rates). Figure 3 gives a path selection pattern design, three paths(Path1, path2 and path 3) would have different path diversity( hops, transmission rate, BER, retransmission count, etc.), UPP decide what portion of OVL image will be transmitted over which path so image quality can be well protected. At the physical-link layer, $\mathrm{AMC}$ and $\mathrm{PC}$ are employed to achieve required BER to protect image transmission (called UBP) as we discussed in the MACPHY design model [10]. We want to determine appropriate
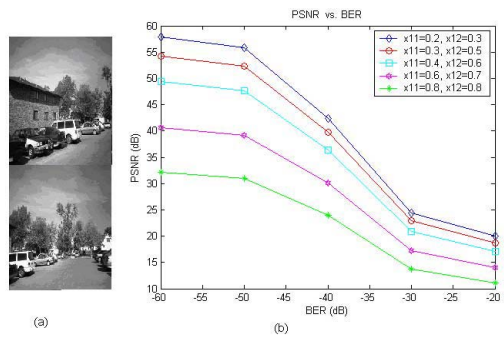

Fig. 2. An example for overlap region transmission diversity by two paths

distribution ratios, and allocate limited resource (Power, rate, etc.) into each node-disjoint path to implement UPP and UBP scheme for image delivery. Corresponding with path diversity,

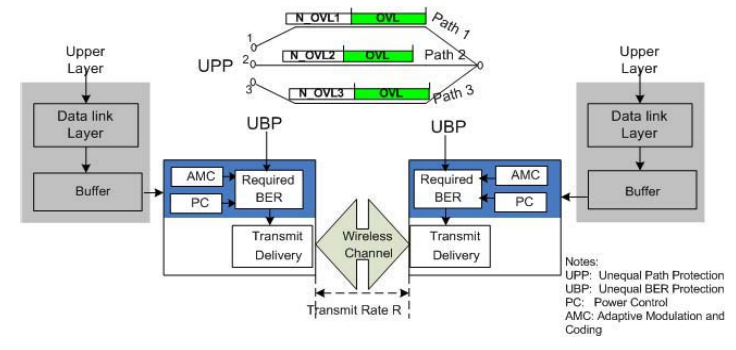

Fig. 3. Unequal path protection and BER protection

the region diversity can be implemented in (2), which indicates that different level thresholds $\left(D_{T}(O V L), D_{T}\left(N_{-} O V L\right)\right)$ can be applied in OVL and N_OVL region for expected image quality. The distortion threshold of OVL region is less than N_OVL regions. With different average image quality threshold requirements, it could potentially provide benefits with respect to energy efficiency for whole system.

$$
\begin{aligned}
& \left.\sum_{i=1}^{M} \overline{D_{i}\left(O V L_{i}\right.}\right)<D_{T}(O V L) \\
& \left.\sum_{i=1}^{M} \overline{D_{i}\left(N \_O V L_{i}\right.}\right)<D_{T}\left(N \_O V L\right)
\end{aligned}
$$

\section{DYNAMIC ROUTING PATH SELECTION AND RESOURCE ALLOCATION WITH DISTRIBUTE RATIOS}

Consideration of distribution ratio with routing path selections (UPP) and resource allocation (UBP) together would offer extra benefits in energy efficiency and sensor life time extensions with required image delivery quality. For example, in Figure 4, senor nodes $(1,2,3,4)$ have equal initial energy except for the cluster head. After a period of time for image transmission, they left 30\%,60\%, 60\% and 30\% remaining energy individually. Correlated image sensors s1 and s2 sense target and capture images being sent to cluster head by two node-disjoint paths. Figure 4 illustrated it by two scenarios: one is that distribute ratio $X$ is determined without dynamic routing path selection, the other is the distribution ratio $X$ is determined with dynamic routing path selection.

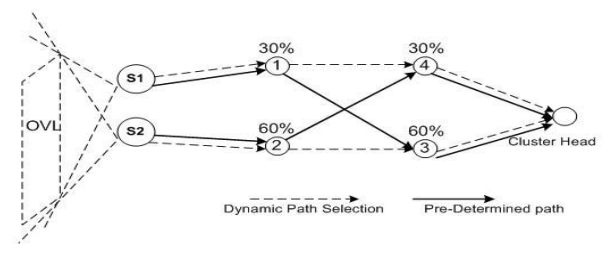

Fig. 4. Dynamic path selection and pre-determined path example

In the first scenario, if the routing path has pre-determined, for example, s1 image sensor has routing path $(\mathrm{s} 1->1->3-$ $>$ cluster head), and s2 image sensor has routing path (s2$>2->4$-cluster head), so the resource (Power, rate) will be allocated on the pre-determined routes. Optimal distribution ratio is determined based on the condition of pre-determined route paths. Once it is available, the source rate of $\mathrm{s} 1$ 
and s2 can be determined. Since each route has to provide transmission rate and PER to support its source rate image delivery quality in this scenario, node 1 has to afford as same transmission load as node 3 because they are at the same route path, so node 1 would die earlier since it just has $30 \%$ residual energy. The same thing will happen on the other route (s2->2->4->cluster head), which makes energy consumption unbalanced and network life time reduced. The problem of unbalanced energy consumption and reduced network lifetime in the first scenario can be solved by dynamic route path selection with distribution ratio, resource-distortion estimation. In this scheme, distribution ratio has to be interdependent with routing path selection. Given a network topology, the routing path selection should also be taken to balance sensor life time as possible as it can. Therefore, a source-rate based routing path selection is well suited to be applied in this scheme. We propose a Multi-Level Rate-Oriented routing (MLRR)scheme based on the link rate assignment, we separate the sensors between the group of source sensors and the cluster head into multiple levels according to their distance to the head. Sensors at each level will be assigned with data rates according to their residual energy. After a sensor determines its rate according to the residual energy level, it will choose the next hop node in the next level based on its rate. The detail are refereed to our work in [9]. This routing selection scheme guarantees that each individual source node in the source group can find nodedisjoint path while satisfying their rate constraints. As shown in figure 4 , for example, MLRR will choose the new path1 $\{\mathrm{s} 1-$ $>1->4->$ cluster head $\}$ for image sensor s1 and path2 (s2->2$>3$->cluster head) for image sensor s2 while being adaptive to appropriate distribution ratio. In this case, more portions of the OVL region will be transmitted by path 2 due to more remaining energy on its relay nodes, and fewer portions of the OVL region will go to path 1 due to less remaining energy. This distribution ratio being adaptive to route path selection offer more gains in sensor life time balancing than scheme in the first scenario.

\section{OPTIMAL IMAGE TRANSMISSION MODEL}

With the transmission diversity for different image regions on multiple paths based on MLRR, we are looking for an optimal solution to achieve higher energy efficiency and better load balancing under the requirement for image transmission quality, i.e., image distortion requirement. Let $S$ be a set of nodes from image sensor groups that can perform cooperative measurements on the target, and $N$ be the total number of nodes in $S$. We also denote by $E_{i},\{i=1,2, \ldots \ldots N\}$ the sensors' residual energy with $i$ as the sensor index. Each N_OVL region is labelled by $N_{-} O V L_{i}$, and $O V L$ region is labelled by $O V L_{j},\{j=1,2, \ldots \ldots N) . M$ is the number of overlap regions in the interest scene. We use $x_{i, j}$ to denote the fraction of the overlap region $O V L_{j}$ that is to be sent by sensor $i$.Therefore, the $i^{t h}$ sensor's lifetime can be expressed as follows:

$$
t_{i}=\frac{E_{i}}{\left(N_{-} O V L_{i}+\sum_{j=1}^{M} x_{i, j} \cdot O V L_{j}\right) \cdot \text { Cost }_{i}}
$$

Where Cost $_{i}$ is the energy consumption per unit when the $i^{t h}$ sensor sends image to the next-hop node, which can be derived from energy model in [10].In the network model, all correlated image sensors should die at the same time to balance the network lifetime to avoid losing the sensing coverage. Therefore, the life time of each image sensor is balanced after we consider the constraint in (4), the remaining question is to how to find an optimal distribution ratio combination to transmit image sensor in unequal way so that the total energy consumption will be minimized.

$$
\begin{aligned}
& \frac{\text { Cost }_{1} \cdot E_{i}}{E_{1} \cdot \text { Cost }_{i}}\left(N \_O V L_{1}+\sum_{j=1}^{M} x_{1, j} \cdot O V L_{j}\right)= \\
& \left(N_{-} O V L_{i}+\sum_{j=1}^{M} x_{i, j} \cdot O V L_{j}\right)
\end{aligned}
$$

For single node-disjoint path, the energy cost $E_{p}$ is the sum of each hop energy consumption. We use the PHY-MAC layer energy model we have developed in [10].

$$
\begin{aligned}
& \bar{E}\left(B E R, L_{D A T A}, R_{C T R L}, R_{D A T A}, P_{D A T A}, R T_{\max }\right) \\
& =\left(\overline{E_{T}}+\overline{E_{R}}\right) \cdot \overline{R T} \\
& E_{\text {total }}=\sum_{P=1}^{N}\left(N_{-} O V L_{p}+\sum_{i=1}^{M} x_{i, p} O V L_{i}\right) \cdot E_{p}
\end{aligned}
$$

the average total energy consumption $\bar{E}$ can be expressed as a function of desirable BER requirement $B E R$, frame length $L_{D A T A}$, control packet transmission rate $R_{C T R L}$, data packet transmission rate $R_{D A T A}$, transmission power $P_{D A T A}$ and retransmission limit $R T_{\max }$ in [10], $N_{-} O V L_{p}+\sum_{i}^{N} x_{i, p} O V L_{i}$ is the amount of image data being transmitted on path $p, E_{\text {total }}$ is total energy consumptions for transmitting all the image regions. There is cross-layer optimization problem that can be expressed to find an optimal set $\left\{x_{i, j}\right\}$ such that

$$
\left\{x_{i, j}^{o p t}\right\}=\underset{x_{i, j}}{\arg \min }\left\{E_{\text {total }}\right\} \text { subject to (2) (4) and } \sum_{i=1}^{N} x_{i, j}=
$$

1.

\section{SimUlation AND ANALYSIS}

In our scheme, due to simple cluster-based topology with few hops, the collaborative optimizations done by cluster head and sink are feasible. By offline learning at sink, the sink has the basic knowledge of region distribution patterns for image sensors. Cluster head collects the initial energy status for each sensor, and creates energy status profile at the base station. It applies the algorithm described in the previous section to calculate the routing and allocate resource based on the topology and energy status information. When the view field of image sensor has been changed, the image sensor raises a flag, and the cluster head or sink can detect the region pattern change by analyzing the content of image block sent from source sensors. The cluster head estimates the residual energy 
based on the image block it received, and updates the residual energy profile for each sensor at base station. Once the region distribution pattern has changed, the cluster head recalculates the optimal result and broadcasts update message to update the rate and route table. We illustrated our solution in following example. The overlapping and no-overlapping regions can be denoted into several areas. We applied same example in figure 1. In Figure 5 we get some results with consideration of image sensor life time balance (These constraints reduce the parameters to only unknown $\mathrm{x} 11$ ). In figure $5(\mathrm{a})$, there is total energy consumption shaking due to life time balance at different distribution ratio with different packet length (same as frame length). Longer frame length 128 has less energy consumption than other frame length (48, 64, and 80). Figure 5 (b) describes a PSNR (image quality) change with different distribution ratio(x11). If we defined image quality requirement $(>28 \mathrm{~dB})$ as shown in figure $5(\mathrm{~b})$, there is an optimal ratio around 0.25 that has minimal energy consumption while satisfying bound requirement as shown in figure 5(a). In our experiment, we use Genetic Algorithm to find an optimal solution with given path diversity parameters and distortion bound. To solve the optimization problem formalized in the previous sections, we design a specific GA, in which Fitness function is defined as $\left(E_{\max }^{\text {total }}-E_{\min }^{\text {total }}\right) /\left(E_{c}^{\text {total }}-E_{\min }^{\text {total }}\right)$, where $E_{c}^{\text {total }}$ is the total energy consumption in the current generation. In our scheme, only a part of unknown $x_{i j}$ need to be coded into genes since others will be accordingly bound by those constraint equations elaborated in section $\mathrm{V}$. This reduces the complexity of computation greatly. For our simulation, part of the viewing field where image sensors capture pictures is illustrated in Figure 2(a). We get simulation results in Figure 6. In Figure 6(a), optimal set achieves more and more energy savings compared with the non-optimal set when the sampling frequency increases (i.e., the source rate increases), and the optimal set spends less energy consumption than Non-optimal set. This performance improvement becomes more obvious, up to $21 \%$ in the simulation, when the source rates of image sensors increase. Figure 6(b) shows that the MLRR scheme with the optimal set based on genetic algorithm also extends the network lifetime considerably by a factor up to $11 \%$. This is because it utilizes the higher residual energy nodes, and balances the node energy consumption in WSN.

\section{CONCLUSIONS}

In this paper, we studied how each correlated image sensor within WSN can transmit appropriate fractions of overlap regions to the base station optimally, and how images can be sent through multiple node-disjoint paths. We found that the effective pattern of collaborative image transmission is not only determined by the network resource of each image sensor itself, but also is related to the network routing hop by hop selection. The image region and path diversity have also been exploited in order to save energy while satisfying image quality bound by applying UPP and UBP schemes to multiple routing paths. Based on it, we formed a network optimization problem with the considerations on characteristics of correlated image sensors, and the energy-aware path selection strategy corresponding with path diversity. The simulation results have shown that our algorithm and procedures achieve considerable gains with respect to the energy efficiency, network lifetime, and improved image qualities in image-sensorbased WSN.
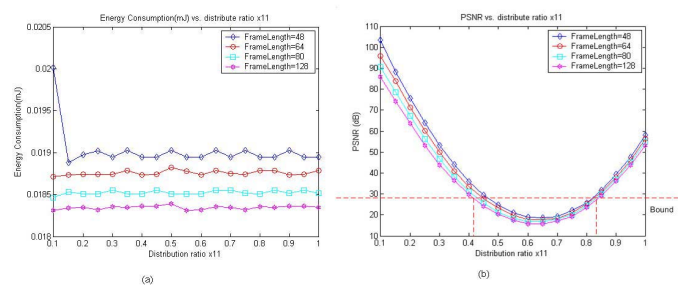

Fig. 5. Energy consumption and PSNR Vs Distribution ratio
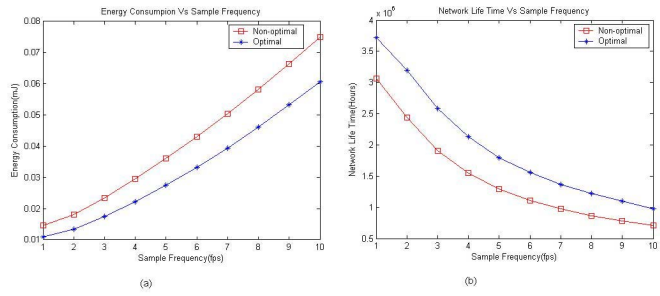

Fig. 6. Energy consumption, Network lifetime Vs Sample frequency

\section{ACKNOWLEDGMENT}

This research project was partially supported by the Nebraska Research Initiative (NRI-2006) grant.

\section{REFERENCES}

[1] J. Chang and L. Tassiulas, "Maximum lifetime routing in wireless sensor networks," IEEE/ACM Trans. Netw., vol. 12, pp. 609-619, Aug. 2004.

[2] S. Back, G. Veciana, and Xun-Su, "Minimizing energy consumption in large-scale sensor networks through distributed data compression and hierarchical aggregation," IEEE JSAC., vol. 22, no. 6, pp. 1130 1140,Aug.2004.

[3] S. Pradhan, J. Kusuma, and K. Ramchandran, "Distributed compression in a dense microsensor network," IEEE Signal Process. Mag., vol. 19, no. 2, pp. 51-60, Mar. 2002.

[4] R. Wagner, R. Nowak, and R. Baraniuk, "Distributed image compression for sensor networks using correspondence analysis and super-resolution," , Int. Conf. Image Processing, vol. 1, 2003.

[5] Z. Xiong, A. Liveris, and S. Cheng, "Distributed source coding for sensor networks," IEEE Signal Process. Mag., vol. 21, no. 5, pp. 80-94,

[6] Z.Wu, A.Bilgin, M.Marcellin, "Joint source/channel coding for multiple images," IEEE Trans. On Communications, vol.53, issue 10, Oct. 2005

[7] L.Qiong, M.van der Schaar, "Providing adaptive QoS to layered video over wireless local area networks through real-time retry limit adaptation," IEEE Transactions on Multimedia, Vol.6, 2004, pp.278-290

[8] S. Mao, S. Lin, S.S. Panwar, Y. Wang, E. Celebi, Video transport over ad hoc networks: multistream coding with multipath transport, IEEE JSAC 21 (5) (2003) 1721-1737

[9] H.Wang. D.Peng, W.Wang, H.sharif, H.chen, "Interplay Between Routing and Distributed Source Coding in Wireless Sensor Network" to appear in IEEE International Conference on Communications 2007

[10] W.Wang, D.Peng, H.Wang, H.Sharif, H.Chen, "Energy Efficient Multirate Interaction in Distributed Source Coding and Wireless Sensor Network," to appear in WCNC 2007, Mar 2007. 